\title{
Hybridization Associated with Cycles of Ecological Succession in a Passerine Bird
}

\author{
Renée A. Duckworth and Georgy A. Semenov \\ Department of Ecology and Evolutionary Biology, University of Arizona, Tucson, Arizona 85721 \\ Submitted November 14, 2016; Accepted April 10, 2017; Electronically published August 16, 2017 \\ Online enhancements: appendix figures and tables. Dryad data: http://dx.doi.org/10.5061/dryad.6dm3c.
}

\begin{abstract}
Aв ST RACT: Identifying the diversity of contexts that can lead to hybridization is important for understanding its prevalence and dynamics in natural populations. Despite the potential of ecological succession to dramatically alter species co-occurrence and abundances, it is unknown whether it directly promotes hybridization and, if so, has long-lasting consequences. Here, we summarize 30 years of survey data across 10 populations to show that in western and mountain bluebirds, heterospecific pairing occurs during repeatable and transient colonization events at the early stages of species turnover. Despite mixed pairing occurring only during early succession, genetic data showed presence of hybrids at both early and late successional stages. Moreover, hybrids showed novel patterns of variation in morphology and behavior, emphasizing that even ephemeral contexts for hybridization can have important evolutionary consequences. Our results suggest that because ecological succession often brings together closely related competitors in disparate numbers but lasts for only a brief period of time, it may be a widespread but underappreciated context for hybridization.
\end{abstract}

Keywords: hybridization, competition, ecological succession, Sialia, population size, species replacement.

\section{Introduction}

Hybridization can be an important factor in evolution because it can lead to speciation, create opportunities for adaptive radiation, and facilitate rapid adaptation to novel environmental conditions (Arnold 1992; Barton 2001; Seehausen 2004); however, its importance will depend on its prevalence. Historically, hybridization among animal species has been thought of as relatively rare and confined to narrow zones of sympatry where two recently diverged species come into secondary contact (Mayr 1963; Mallet 2005; Schwenk et al. 2008). Yet recent studies using genomic techniques show that historical signa-

\footnotetext{
* Corresponding author; e-mail: rad3@email.arizona.edu. ORCIDs: Duckworth, http://orcid.org/0000-0003-0427-4108.

Am. Nat. 2017. Vol. 190, pp. E94-E105. (C) 2017 by The University of Chicago. 0003-0147/2017/19004-57384\$15.00. All rights reserved. DOI: $10.1086 / 693160$
}

tures of hybridization are widespread (Mallet et al. 2016); moreover, the discovery of mosaic hybrid zones - where hybridization occurs throughout a species range at habitat transitions (Rand and Harrison 1989; Emms and Arnold 1997; Larson et al. 2013) — indicates that hybridization is not always confined to narrow zones of secondary contact. In addition to variation in the spatial context of hybridization, there is also substantial temporal variation because some hybrid zones are highly stable over time and others are relatively ephemeral, occurring at a particular location only briefly during range expansion or habitat changes (Pearson and Rohwer 2000; Robbins et al. 2014). Such ephemerality make these hybrid zones difficult to detect and may lead to an underestimation of the occurrence of hybridization. Identifying specific ecological contexts in which hybridization is likely to occur can reduce this underestimation problem, enabling a more proactive assessment of hybridization's prevalence.

It has long been recognized that hybridization is more likely to occur when one species is rare (Hubbs 1955; Randler 2002), and many examples of hybridization are from recent colonization events in which species are expanding their range either naturally or through human introduction (Abbott et al. 2003; Rieseberg et al. 2007; Currat et al. 2008; Senn and Pemberton 2009; Taylor et al. 2014). When an invading species spreads into an area already occupied by a related species, the invading species is initially rare (Rheindt and Edwards 2011), thus increasing the likelihood of heterospecific mating because of low availability of conspecific partners. Another context that influences relative species' abundances and leads to hybridization is habitat variability. In patchy environments, habitat variation affects the opportunity for species to interact, and hybridization can produce a mosaic of introgressed and purebred populations (Rand and Harrison 1989). This is thought to reflect the adaptation of each parent species to a different habitat type; thus, hybridization occurs in the areas of habitat transitions, which often show disparity in the species' relative abundances (Jansson et al. 2007; Lepais et al. 2009; Larson et al. 2013). Finally, the propensity for hybrid- 
ization to occur when one species is rare has long posed challenges to conservationists because it potentially threatens the integrity of threatened and endangered populations (Rhymer and Simberloff 1996; Burgess et al. 2005).

Ecological succession - where there is a change in the species composition of a community over time - often produces a disparity in population sizes of closely related species and thus may be an important context for ephemeral hybrid zones. Typically, succession occurs in ecosystems that experience periodic disturbance (Platt and Connell 2003). Disturbance results in a sudden shift in habitat type that often removes species in prior residence, providing a clean slate for recolonization (Levin and Paine 1974). Newly disturbed habitat is usually colonized first by the most dispersive species, and over time, less dispersive species that are better competitors invade and begin to replace earlier-arriving species (Yu and Wilson 2001; Cadotte et al. 2006; Cadotte 2007). Thus, ecological succession is characterized by predictable transitions in the relative abundance of different species over time (Platt and Connell 2003), providing an opportunity for hybridization, particularly if the species that dominate during different successional stages are close relatives; yet hybridization has rarely been explicitly directly linked to ecological succession dynamics (Robbins et al. 2014). Direct observations of heterospecific pairing during ecological succession are needed to explicitly link the emergence of cross-species mating to distinct stages of ecological succession.

Here we present evidence of heterospecific pairing between mountain and western bluebirds (Sialia currucoides and Sialia mexicana) during early stages of successional replacement and show that its impacts are potentially widespread because it has consequences for a variety of phenotypic traits and leads to signatures of hybridization and introgression across populations at varying stages of colonization. Where the two bluebird species' ranges overlap in the northwestern United States, they compete for nest cavities in successional postfire habitat (Duckworth 2014). Differences in competitive ability and dispersal propensity produce cycles of species replacement that result in predictable changes in the relative abundances of these species over time, with mountain bluebirds (the more dispersive species) colonizing newly created habitat patches first but eventually being replaced by the slower to arrive but more aggressive and competitively dominant western bluebirds (Duckworth and Badyaev 2007; Duckworth 2012; Duckworth et al. 2015). In this article, we have three main goals. First, we use an extensive survey of breeding populations spanning 15 years $(N=1,297$ pairs/2,594 individuals) to test the idea that heterospecific pairing between western and mountain bluebirds occurs only in early successional populations. Second, we use molecular markers to verify that heterospecific pairings result in hybrid offspring. Finally, we determine the potential evolutionary importance of such ephemeral hybridization by assessing whether intro- gression is ongoing and whether it has important phenotypic consequences.

\section{Methods \\ Study Populations and General Sampling}

Ten populations of known history and age were surveyed in western Montana from 2001 to 2015 (for details of population location, years sampled, and numbers of breeding pairs observed at each site, see fig. 1). We also reviewed the literature for historical observations of mixed pairing between these species. Because all of the sites were originally dominated by open meadows that lacked natural nest cavities, suitable habitat was created with the addition of nest boxes, and bluebirds' colonization of the habitat occurred in the spring following nest box placement. This placement of nest boxes produces patterns of species succession that are similar to natural postfire habitat in that mountain bluebirds colonize both nest box and natural habitat at high densities in the first year, whereas western bluebirds are delayed in their arrival, but once they colonize, their population numbers typically increase linearly (Saab et al. 2007; Hutto and Patterson 2016; Duckworth et al. 2017). Thus, the proposed key factor that leads to hybridization during early successional stages (a disparity in species number) is mirrored in newly created nest box populations.

At each site during sampling years (fig. 1), we visited nest boxes during May, June, and July to determine species identity of nesting pairs. Successional stage of sites was categorized as early if western bluebirds comprised $<33 \%$ of breeding bluebird pairs and late if they comprised $>33 \%$. Disparity in species numbers was used to categorize populations because this is the key factor hypothesized to lead to heterospecific pairing. Through our long-term sampling effort, we observed 1,297 breeding pairs across 10 populations that were monitored during early $(N=5)$ or late $(N=7)$ successional stage (two populations were monitored across both stages).

We visited a subset of nests at least weekly to monitor the progress of nests, collect blood samples from nestlings, and measure aggressive behavior. A subset of resident adults were captured at each site using traps baited with mealworms to collect a blood sample for molecular analysis, mark them with a unique color band combination, and take standard morphological measurements, including body mass and length of the tarsus, tail, wing, and bill. Adults that were not banded as nestlings were aged using validated feather wear indices (Shizuka and Dickinson 2005). Breast plumage of adults was photographed using a digital 35-mm camera in a standard position. Birds were placed in a standardized position on the dorsal side, with the anterior point of the beak held in place (see fig. A1; figs. A1-A3 are available online). Photographs were taken at a constant distance against a neutral gray background with scale markings. Mountain bluebirds lack a phaeomelanin 


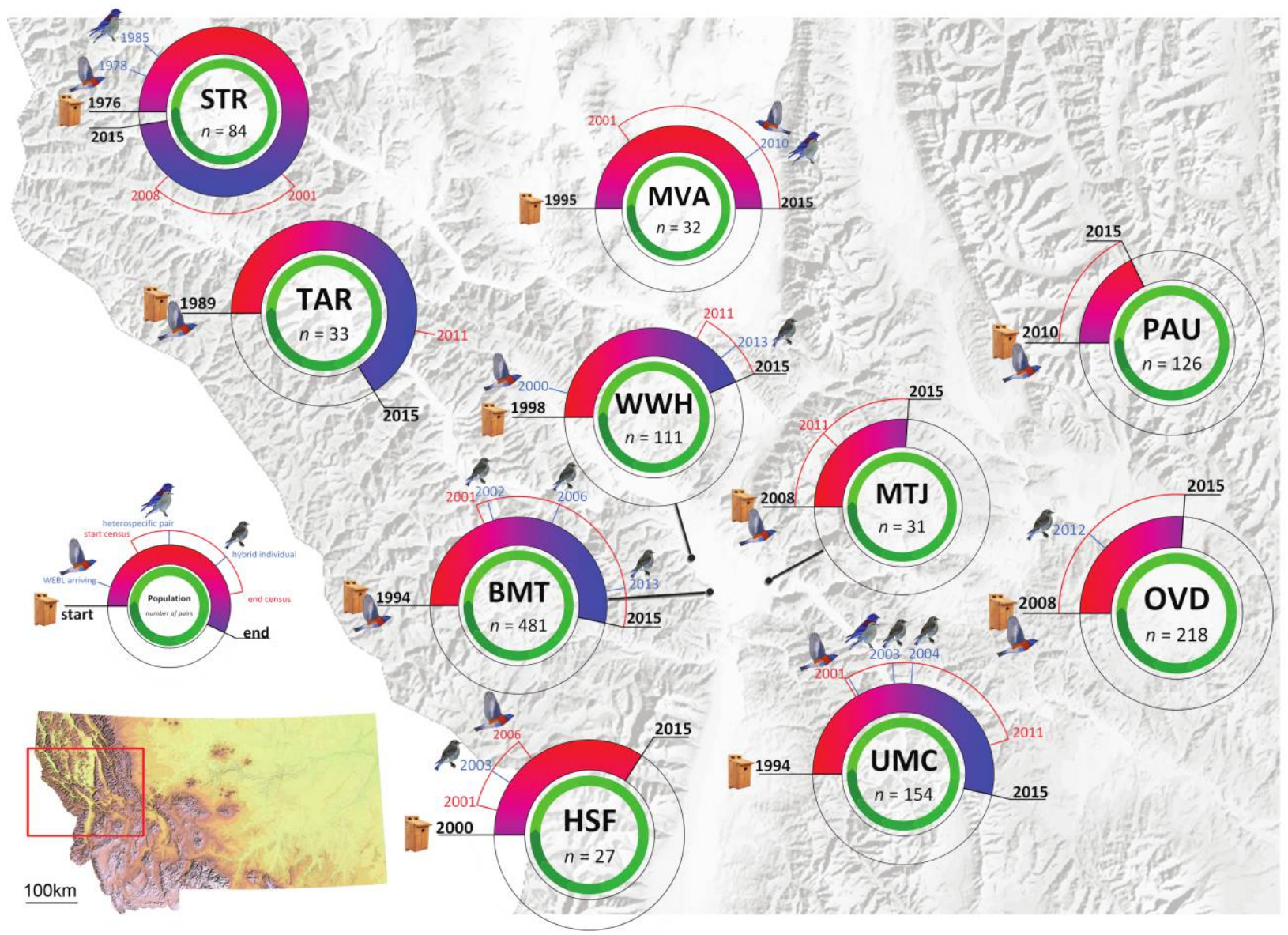

Figure 1: Observations of heterospecific pairing and hybrid adults in 10 populations that vary in successional stage across western Montana. New habitat was produced using nest boxes placed in open meadows. Outer ring of circles indicates current successional stage: populations are colonized first by mountain bluebirds, which are eventually replaced by aggressive dispersing western bluebirds, which in turn are replaced by nonaggressive philopatric western bluebirds. Shading from red to purple indicates changes in bluebird aggression, with warmer colors indicating higher aggression periods. Inner green ring indicates the predictable increase in western bluebird breeding density across the successional cycle. Blue lines indicate heterospecific pairs and hybrid adults, and red lines indicate sampling period. Information inside circles indicates population name (STR, St. Regis; TAR, Tarkio; MVA, Moiese Valley; WWH, Water Works Hill; BMT, Blue Mountain; HSF, Hamilton; MTJ, Mount Jumbo; UMC, Upper Miller Creek; PAU, Paws Up; OVD, Ovando) and sampling effort as number of pairs observed in each population (for details, see table 1). Inset map shows region sampled in western Montana. Hybrids were observed in both early and late successional stage populations, while heterospecific pairing was observed only in early successional populations.

breast patch; thus, we measured only western bluebirds and hybrids that had a clearly delineated breast patch (fig. A1). We used ImageJ (Abramoff et al. 2004) to measure the area of breast patch in square centimeters. Each measurement was averaged among three replicates.

We measured aggression of all breeding adults at the Blue Mountain site during all years of the study, and we sampled a subset of adults at the other sites across years using standardized protocols that simulate a territorial intrusion by a heterospecific competitor for nest sites, the tree swallow Tachycineta bicolor (for details, see Duckworth 2006a). Bluebird's aggressive response toward a heterospecific competitor is highly correlated with response toward a conspecific competitor and is related to resource holding potential (Duckworth 2006a, $2006 b$ ). Tree swallows were used because they are the most frequent nest site competitor of bluebirds at all of the sites, and use of a nonbluebird species allows us to acquire a measure of aggression that is independent of the variable history of overlap of the two bluebird species across sites (Duckworth and Badyaev 2007). Moreover, this assay standardizes responses among the sexes (Duckworth 2006b). To avoid pseudoreplication, different tree swallows were used each day to limit the number of times the same swallow was used to measure aggression for different bluebirds. Moreover, previous work has shown that there is no effect of swallow identity on bluebird aggression (Aguillon and Duckworth 2015). To 
conduct aggression trials, we placed a live tree swallow at a focal male's nest box and recorded responses over a 2-min period concurrently by an observer and a camera. Observers noted the number of times an individual flew by, attacked, and hovered, and aggression scores were assigned using a weighted count of the behaviors, where each hover was assigned 1 point, each flyby 2 points, and each attack 3 points. Counts of these behaviors by the real-time observer were independently verified by a separate observer from the video recordings.

\section{Detection of Hybrids and Molecular Methods}

We selected 35 and 32 presumed purebred western and mountain bluebirds, respectively, that were distributed across the study populations and years of study (table A2; tables A1A3 are available online; data deposited in the Dryad Digital Repository: http://dx.doi.org/10.5061/dryad.6dm3c [Duckworth and Semenov 2017]). Whenever possible, we included adults that were captured in both their first year of breeding, which is their second year of life (second-year [SY] birds), and again at least one subsequent year (after-second-year [ASY] birds). This allowed us to control for age-related variation in wing and tail length in these species (Power and Lombardo 1996; Guinan et al. 2000). We also preferentially included individuals that had both morphological and behavioral data available. We included six (bands 21344, 21345, 21325, 21326, 93528, and 93529) of the 10 offspring of heterospecific pairing in the admixture analyses because including a higher number of related individuals would have biased allele frequencies and distorted the results. Therefore, to determine how many of the offspring were true offspring of heterospecific pairs and thus hybrids, we assessed their match to parents' and siblings' genotypes using the parentage and sibship method implemented in Colony 2.0.5.9 (Wang and Santure 2009). We had genetic data for only one of the adult pairs, so for the other nest we compared only siblings, assessed their status as full or half-sibs, and - on the basis of this assessment and their hybrid status - inferred whether they were offspring of the social pair or of an extrapair mating. Our admixture analyses also included one individual that was flagged as unusual in morphology (20847) on the basis of observations during banding, the male (93524) and female (93516) of the Upper Miller Creek (UMC) heterospecific pair, and two males that we previously identified as either the extrapair mate (75259; Duckworth 2006c) or a subsequent social mate (63911) of the hybridizing female in the UMC population.

To verify that heterospecific matings resulted in the production of hybrids and to evaluate the hybrid status of adults, we genotyped individuals using fluorescently labeled primers to amplify 15 microsatellite loci (SMEX1, SMEX2, SMEX4, SMEX5, SMEX6, SMEX7, SMEX8, SMEX9, SMEX10, SMEX11, SMEX13, SMEX14, CUU02, CUU04, and SSI8; Ferree et al. 2008; Duckworth and Kruuk 2009). Primers were chosen on the basis of evidence for successful amplification in all samples, presence of allele length polymorphism, and biallelic inheritance (see table A3 for allele frequencies; data deposited in the Dryad Digital Repository: http://dx.doi.org/10.5061 /dryad.6dm3c [Duckworth and Semenov 2017]). A subset of the primers (up to nine, depending on the year of study and resolution needed) was used previously for paternity analysis (Duckworth and Kruuk 2009), and additional primers were added for this study to increase our power to detect hybrids. These primers were not previously known to differ across species (because they were developed for running paternity analyses within species), and in this study we ran simulation analyses to verify that they could be used to identify hybrids. DNA was extracted from blood samples using a $5 \mathrm{M}$ salt solution (Miller et al. 1988). Polymerase chain reaction product was analyzed using an ABI 3730 DNA Analyzer at the University of Arizona Genetics Core. Alleles were scored using the Microsatellite Plugin in Geneious 6.0 (Kearse et al. 2012 ). Only genotypes with $<13 \%$ of missing data were included in analyses.

\section{Statistical Analysis}

To assess whether hybridization occurs more frequently during the early successional stage, we used the Fisher exact test. This test is appropriate for this analysis because we are testing a hypothesis about categorical data that results from classifying our successional stages in two different ways (early vs. late) and seeing whether this classification is associated with heterospecific pairing.

We used Bayesian clustering methods implemented in NewHybrids 1.1 Beta (Anderson and Thompson 2002) and STRUCTURE 2.3.4 (Pritchard et al. 2000) to assess genetic evidence for hybridization and introgression. STRUCTURE assignment scores $(q)$ indicate the probability of an individual belonging to each of $K$ genetic clusters and can also be roughly interpreted as a proportion of ancestry derived from each of these clusters. The method implemented in NewHybrids estimates probability of an individual (also denoted as $q$ ) belonging to several genotype classes, including purebreds, hybrids, and backcrosses, which can be configured by the user. These two methods are considered as complementary for detection and validation of individuals with admixed origin as STRUCTURE in general shows higher power and NewHybrids provides higher accuracy of hybrid assignment (Burgarella et al. 2009).

An important aspect of delineating between purebreds and hybrids when using Bayesian assignment methods is the appropriate estimation of the optimal threshold values $\left(T_{q}\right)$ associated with corresponding $q$ scores (Vaha and Primmer 2006). For both assignment methods we initially used $T_{q} \geq$ 0.9 (according to criterion 3 of Burgarella et al. 2009) to eliminate potentially admixed individuals from the set of ge- 
notypes chosen to simulate parental data sets. Then, alleles were randomly drawn from the pool of 31 Sialia mexicana and 31 Sialia currucoides genotypes to create 100 simulated genotypes of each species as well as $100 \mathrm{~F}_{1}$ hybrids and two first-generation backcrosses using HYBRIDLAB 1.1 (Nielsen et al. 2006). These simulated genotypes were then analyzed in NewHybrids and STRUCTURE.

For the STRUCTURE analysis we used $K=2$ because our preliminary analyses indicated that the most likely number of clusters is 2 ( $K$ tested from 1 to 4 ; fig. A3). We ran the analysis using an admixture model, correlated allele frequencies, and 500,000 burn-in followed by 1,000,000 Markov chain Monte Carlo (MCMC) iterations with five replicates. We ran the NewHybrids analysis for five replicates using Jeffrey-type priors for mixing proportions and allele frequencies with 100,000 burn-in followed by 200,000 MCMC iterations, after verifying that the results do not significantly differ for a higher number of burn-in and MCMC values. For the NewHybrids analysis we ran two sets of genotype classification. The first consisted of five genotype classes: two purebreds, first-generation hybrids, and two first-generation backcrosses. The second represents the most conservative approach and includes only three genotype categories: two purebreds and first-generation hybrids. No prior species information was used in both STRUCTURE and NewHybrids. The estimated uncertainty in cluster memberships among runs was summarized using CLUMPP 1.1.2 (Jakobsson and Rosenberg 2007).

For both clustering methods, we tested $T_{q}$ ranging from 0.95 to 0.50 with the 0.05 step. The Bayesian clustering approach performed on 500 simulated genotypes in STRUCTURE resulted in clear separation between parental species with no overlap in q variation limits. The optimal $T_{q}$ that maximized both power and accuracy of assignment was 0.8 (table A1). With this threshold, $90 \%-95 \%$ of simulated parental genotypes were assigned to corresponding categories, and $87 \%$ of hybrids in all categories were identified. The assignment of $80 \%-86 \%$ of parental species and $95 \%$ of all hybrids was correct, suggesting high accuracy. Although $16 \%-23 \%$ of backcrosses had $q$ values overlapping with parental species, only two parental genotypes (1\%) overlapped with $\mathrm{F}_{1}$ hybrids in $q$ scores (fig. A2).

The clustering algorithm of NewHybrids showed high assignment power and accuracy when using three genotype classes. More than $90 \%$ of parental genotypes and $83 \%$ of all hybrids were correctly assigned under the optimal $T_{q}=$ 0.95 (table A1). However, it showed a weak ability to distinguish between simulated parental genotypes and backcrosses (hybrid categories with five genotype classes; table A1). We thus used the assignment procedure with three genotype classes for analysis with NewHybrids. Overall, assignment tests with simulated genotypes indicated consistency between STRUCTURE and NewHybrids algorithms, suggesting high power and accuracy of delineation between first-generation hybrids and both parental species. We thus used $T_{q}$ values and program settings described above for analysis of empircal data.

To assess whether adult hybrids differed in morphological or behavioral traits from adults of either of the parent species, we used mixed models (PROC MIXED in SAS, ver. 9.4; SAS Institute, Cary, NC) that included species status (western, mountain, or hybrid) as a fixed effect. We tested for normality using a Shapiro-Wilk test and confirmed that all variables were normally distributed (all $P>.10$ ), except for the aggression score, which differed significantly from a normal distribution in mountain bluebirds $(W=0.84, P=.019)$ but not in western bluebirds and hybrids (western: $W=$ $0.93, P=.131$; hybrid: $W=0.84, P=.080)$. Given this, we used nonparametric tests for examining species' differences in aggression. Sexes differed in wing length, tail length, body mass, and breast patch size (all $P<.01$ ), so we included sex as a fixed effect in the models for these traits. For birds measured in more than 1 year of the study, we used mean values for aggression score, bill length, and tarsus length because these are highly repeatable across years (for repeatability of aggression, see Duckworth and Sockman 2012; bill: $R=0.92, F=4.25, P<.001$; tarsus: $R=0.99, F=$ 34.84, $P<.001)$. However, for the other morphological traits, ASY birds had longer wings and tails than SY birds for both mountain (wing: $F=14.13, P=.001$; tail, $F=$ 4.92, $P=.037$ ) and western (wing: $F=14.32, P<.001$; tail: $F=8.29, P=.007)$ bluebirds and a larger body mass for mountain $(F=15.87, P<.001)$ but not western $(F=$ $0.05, P=.821)$ bluebirds. Moreover, in a subset of individuals for which we had multiple measures of breast patch size across years, we found that there was a tendency for breast patch size to increase with age (paired $t$-test: $t=2.54, P=$ .06). Thus, for analysis of wing, tail, and breast patch size, we included age as a fixed effect for birds captured as both SY and ASY and included measurements for both ages. We also included individual identity as a random effect in these models to account for the inclusion of the same individual across age classes. For analysis of body mass, we used the same model but also included the interaction between age and species because the age effect on body mass was limited to mountain bluebirds. We used the Dunnett adjustment for multiple comparisons to calculate significance levels for post hoc analysis of differences between hybrids and parent species.

\section{Results}

\section{Heterospecific Pairing in Newly Colonized Populations}

We documented three cases of heterospecific pairing in populations where western and mountain bluebirds overlapped: two in populations we have monitored over the past 15 years 
Table 1: Survey data used to assess the occurrence of heterospecific pairing in populations at distinct successional stages

\begin{tabular}{llcccc}
\hline Site & Stage & $\begin{array}{c}\text { No. years } \\
\text { surveyed }\end{array}$ & $\begin{array}{c}\text { Western } \\
(\%)\end{array}$ & No. pairs & $\begin{array}{c}\text { Heterospecific } \\
\text { pair }\end{array}$ \\
\hline UMC & Early & 4 & 11.3 & 34 & Yes \\
MVA & Early & 4 & 2.0 & 32 & Yes \\
STR & Early & 1 & 30.0 & $15(\max )$ & Yes \\
HSF & Early & 4 & 8.3 & 27 & No \\
OVD & Early & 6 & 14.5 & 218 & No \\
BMT & Late & 15 & 99.4 & 481 & No \\
UMC & Late & 11 & 78.6 & 120 & No \\
WWH & Late & 5 & 63.7 & 111 & No \\
PAU & Late & 5 & 51.6 & 126 & No \\
MTJ & Late & 4 & 66.4 & 31 & No \\
STR & Late & 4 & 89.9 & 84 & No \\
TAR & Late & 1 & 97.0 & 33 & \\
\hline
\end{tabular}

Note: Upper Miller Creek (UMC) was surveyed across both successional stages, and data from St. Regis (STR) during early succession were from Aylesworth 1987. MVA, Moiese Valley; HSF, Hamilton; OVD, Ovando; BMT, Blue Mountain; WWH, Water Works Hill; PAU, Paws Up; MTJ, Mount Jumbo; TAR, Tarkio.

${ }^{a}$ Only estimates of sampling numbers could be inferred.

and one from the literature that occurred in a nest box population that was at an early successional stage more than 30 years ago (Aylesworth 1987), which we monitored during the late successional stage from 2001 to 2006 (table 1). In all three cases, western bluebirds had only recently colonized (mean years since initial colonization $=4.67 \pm 2.73$ ) and comprised less than one-third of the breeding pairs relative to mountain bluebirds (mean percent of western bluebird breeders $=16.83 \% \pm 6.71 \%$; fig. 1 ; table 1 ). The occurrence of heterospecific pairing only in early successional stages differed significantly from random, with three out of five occurrences during early stages and zero out of seven occurrences in late stages (Fisher exact test: $P=.045$ ). In all cases, the male was identified as a western bluebird, and the female was identified as a mountain bluebird on the basis of plumage characteristics.

\section{Evidence of Hybrid Individuals at Different Population Stages}

Both STRUCTURE and NewHybrids consistently identified 10 interspecific hybrids, STRUCTURE identified an additional three, and NewHybrids identified an additional five individuals of hybrid origin (fig. 2). Individuals of hybrid origin were identified in five populations (fig. 1) across all successional stages, including populations of mixed species composition as well as populations comprised of only mountain or only western bluebirds at the time of sampling (fig. 1).

Genetic testing of the offspring in two heterospecific social pairs revealed a high level of extrapair paternity. For the UMC heterospecific pair, only one of four offspring was sired by the social partner. Genotypes of the other three offspring matched a neighboring male (75259) confirmed to be a purebred mountain bluebird (fig. 2). The only genetic offspring of this social pair (93528) was identified as a purebred western bluebird by both STRUCTURE and NewHybrids. Interestingly, the female in this social pair was identified as a mountain bluebird on the basis of plumage but was identified as a hybrid in our genetic analysis (figs. 2, A1). Given this, one of the offspring (93529; STRUCTURE $q=0.478$, NewHybrids $q=0.96)$ between this female and her extrapair purebred mountain bluebird mate (75259) was categorized of hybrid origin according to both methods (fig. 2). The fact that none of the other offspring scored as a hybrid, despite the female of the pair's hybrid status, is consistent with our simulations that showed only a limited ability to discriminate between purebreds and backcrosses on the basis of the available set of loci and indicates that the number of introgressed individuals is more likely to be underestimated than overestimated in our data set. The hybrid female 93516 from this population returned to breed at the study site in 2004 and 2005, but her male social partner did not. In 2004, she paired with a male (63911) that was identified as a mountain bluebird during banding but that genetic testing revealed to be of hybrid origin (fig. 2). We did not have genotype data available for her 2005 mate, but during banding this male was also identified as a mountain bluebird. Of the six offspring produced by the Moiese Valley (MVA) heterospecific pair, two were identified as hybrids (21326: STRUCTURE $q=0.764$, NewHybirds $q=0.96 ; 21344$ : STRUCTURE $q=0.868$, NewHybirds $q=0.99$; fig. 2 ), and four were identified as purebred mountain bluebirds presumably because they were the result of an extrapair mating between the mountain bluebird female and another purebred mountain bluebird male in the population. 


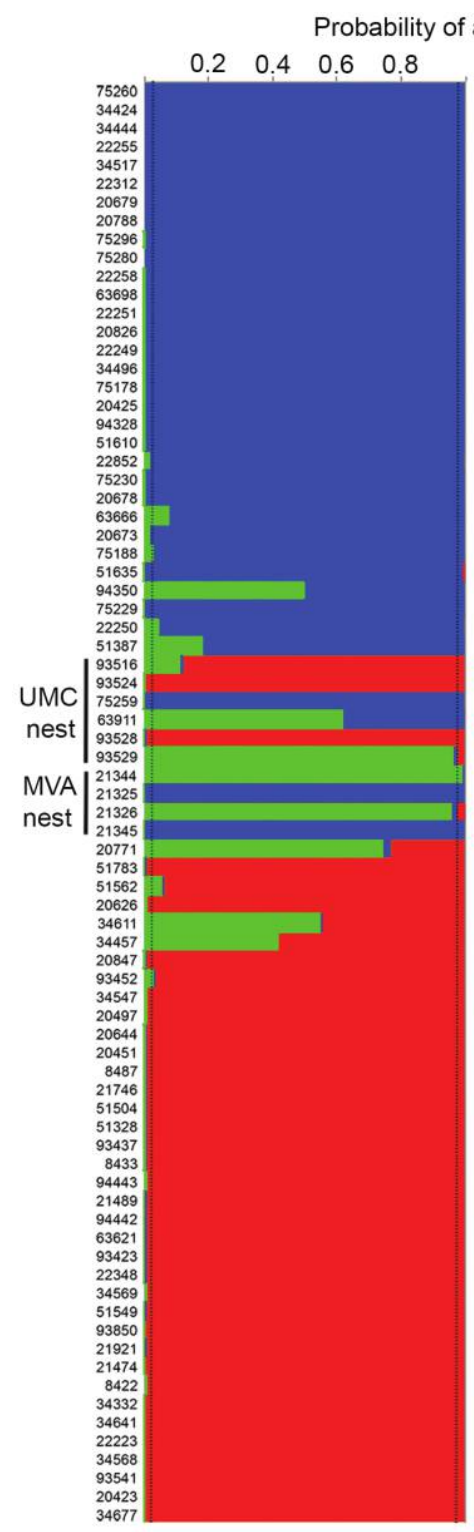

(A)

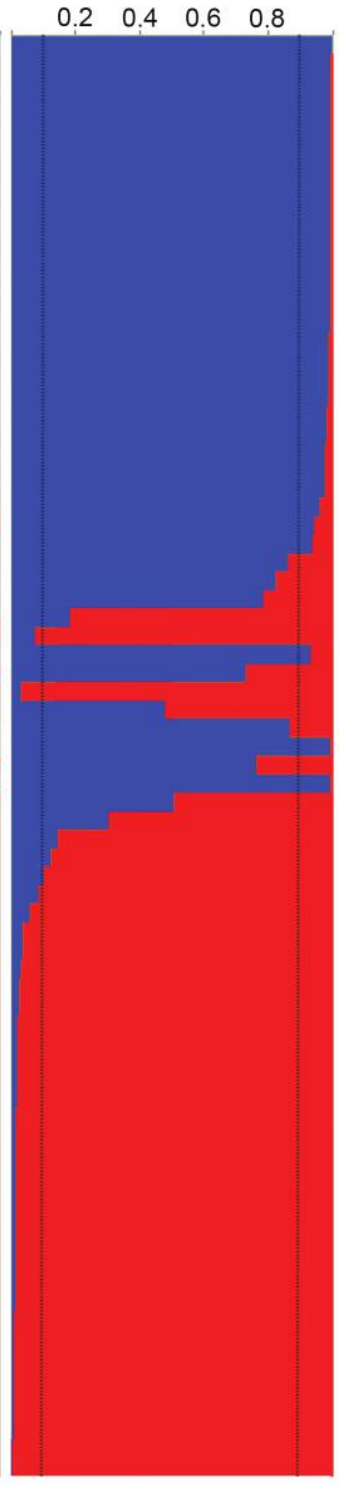

(B)

Figure 2: Results of population assignment tests in NewHybrids with three genotypic classes $(A)$ and STRUCTURE at $K=2(B)$. Each bar is an individual labeled with last five digits of US Fish and Wildlife Service band. Bars indicate probability an individual belongs to western bluebird (red), mountain bluebird (blue), or first-generation hybrid (green) cluster. Dashed lines indicate optimal thresholds used for delineating between purebreds and hybrids. Heterospecific pair members and their offspring indicated on left. UMC, Upper Miller Creek; MVA, Moiese Valley.

\section{Effects of Hybridization on Phenotypic Traits}

Mountain bluebirds were larger in all structural traits compared with western bluebirds $(P<.05$ for all traits; fig. 3), and adult hybrids were generally intermediate in morpho-

logical traits to the parent species. Specifically, in wing and tarsus length, hybrids were smaller than mountain bluebirds and larger than western bluebirds (wing length, overall model: $F=22.01, P<.0001$; post hoc tests: hybrid vs. western, $t=-3.35, P=.007$; hybrid vs. mountain, $t=2.52, P=$ .039 ; tarsus length, overall model: $F=17.66, P<.0001$; post hoc tests: hybrid vs. western, $t=-2.20, P=.054$; hybrid vs. mountain, $t=2.68, P=.017$; fig. $3 B, 3 C$ ). However, in tail length, hybrids were smaller than mountain bluebirds (overall model: $F=13.25, P<.001$; post hoc test: $t=2.64, P=.031$ ) but did not differ significantly from western bluebirds (tail: $t=-1.59, P=.21$; fig. $3 D$ ). Moreover, hybrids did not differ significantly from either parent species in bill length (overall model: $F=5.37, P=.007$; hybrid vs. western: $t=-1.20, P=.36$; hybrid vs. mountain: $t=-1.46, P=.24$; fig. $3 A$ ) or body mass (overall model: $F=9.32, P=.003$; post hoc tests: hybrid vs. western, $t=1.60, P=.21$; hybrid vs. mountain, $t=-1.87$, $P=.13$; fig. $3 E$ ).

Visual inspection of the purebred and hybrid half-sibs from the MVA heterospecific pair showed that they differed in blue coloration, with the purebred showing the more blue-green color of a mountain bluebird and the hybrid having a deeper blue more similar to a western bluebird (fig. 4). We were able to measure breast patch size on five out the 11 adult hybrids with photographs available (for photos of all hybrids, see fig. A1) and did not find any significant differences in mean patch size between western bluebirds and hybrids, although there was a tendency for hybrids to have larger breast patches $(t=1.57, P=.136$; fig. $3 F)$. Given our small sample of introgressed adults, we had low power to detect differences (power $=0.21$ ), and so the possibility that hybrids have larger patches than purebred western bluebirds cannot be ruled out.

Aggression score differed among the species' categories (overall, Kruskal-Wallis test: $\chi^{2}=6.52, P=.038$ ) because mountain bluebirds and hybrids were less aggressive than western bluebirds (Wilcoxon two-sample test: mountain vs. western, $Z=-2.08, P=.038$; hybrid vs. western, $Z=$ $-2.02, P=.021$ ) and more similar to each other (mountain vs. hybrid: $Z=-0.429, P=.668$; fig. 5).

\section{Discussion}

Historically, hybridization was viewed as a rare and unnatural phenomenon that was largely associated with humaninduced habitat changes (Anderson 1949). More recently, it has become accepted that hybridization, if not a common occurrence within taxa, is relatively widespread among taxa, occurring in an estimated 25\% of flowering plants and 10\% of animals (Grant and Grant 1992; Mallet 2005). However, these estimates are potentially a minimum because they are 

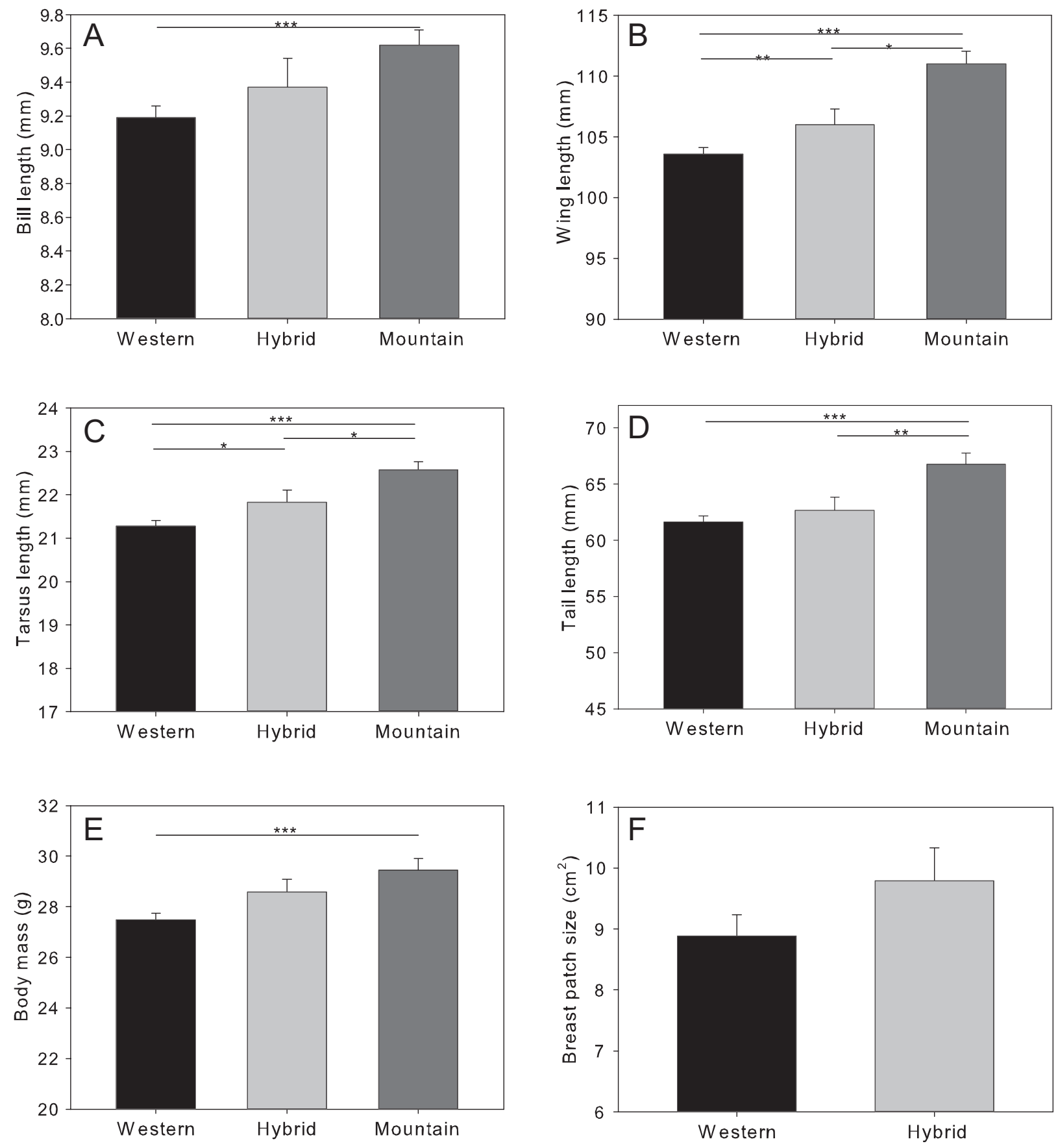

Figure 3: Comparisons of morphological traits (mean \pm SE) among adult Sialia mexicana (western bluebird), Sialia currucoides (mountain bluebird), and hybrids. One asterisk, $P \leq .05$; two asterisks, $P \leq .01$; three asterisks, $P \leq .001$.

typically based on documented occurrences of hybridization that happened to be noticed rather than a systematic survey of overlapping populations of closely related taxa (Grant and Grant 1992). On the other hand, a more recent review controlling for study bias estimates a much lower percentage of 1\% hybridization across all animals (Schwenk et al. 2008).
Thus, the prevalence of hybridization across taxa is still an open question, especially in taxa that are less well studied or where the dynamics that lead to heterospecific mating are ephemeral and difficult to observe.

Identifying the ecological contexts that promote hybridization is thus important because it enables us to better as- 


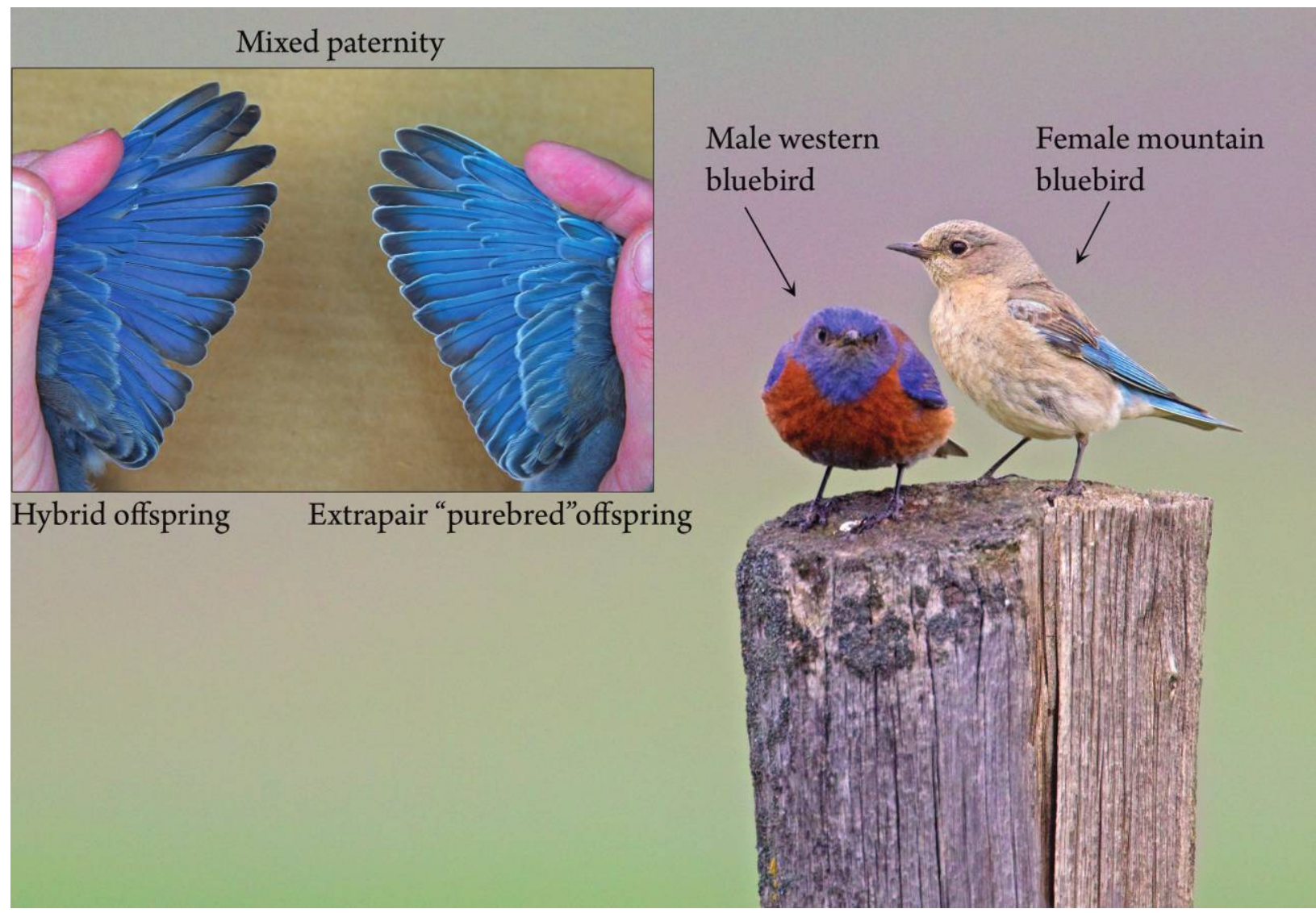

Figure 4: Heterospecific pair from the Moiese Valley population and their offspring. In all hybridizing pairs, the male was a western bluebird and the female was a phenotypic mountain bluebird. Inset shows two half-siblings, 21326 (left) and 21325 (right), one within pair and one extrapair, from this pair's first nest, captured on the day of fledge. The hybrid offspring was fathered by the social mate (western bluebird) and shows a deeper blue coloration than the extrapair offspring that was fathered by a mountain bluebird male. Photo by A. V. Badyaev.

sess its frequency and prevalence and also to understand its evolutionary dynamics. Hybrid zones that occur at stable parapatric species boundaries will have different evolutionary consequences than hybrid zones that are more dispersed spatially (Rand and Harrison 1989). Here, we identified ecological succession as an ephemeral context for the emergence of hybridization between two species of passerine birds. Using survey data from nearly 1,300 breeding pairs over 15 years across 10 populations that varied in successional stage, we found that western and mountain bluebirds are more likely to form heterospecific pairs in the early stages of ecological succession, when western bluebirds have only recently colonized and mountain bluebirds are more common. We also found that hybrids between these species are fertile, resulting in ongoing introgression. Thus, even though heterospecific pairing was confined to early stages of colonization, it has potentially important evolutionary consequences because individuals of hybrid origin were observed across both early and late stages of colonization (fig. 1). Moreover, western and mountain bluebirds overlap over a large part of their breed- ing ranges, and so there is the potential that introgression across this species' range is widespread.

Our study raises the question of why hybridization between these species occurs only at early successional stages - after all, there is also a disparity in species prevalence at late stages of succession, when western bluebirds are common and mountain bluebirds are rare (Duckworth and Badyaev 2007; Duckworth et al. 2017), yet no cases of heterospecific pairing occurred in late successional populations (table 1). One possibility is that western bluebirds' dominance over mountain bluebirds in competition for space and nest cavities influences female mate choice decisions (Duckworth and Badyaev 2007; Duckworth 2014). Competition for nest sites among cavity nesting species is fierce (Duckworth 2014). Thus, early in the successional cycle, female mountain bluebirds may choose a heterospecific mate with a nest cavity rather than forego breeding altogether. Females may then seek extrapair copulations to avoid producing hybrid offspring, a strategy similar to that employed by collared flycatcher (Ficedula albicollis) females (Veen et al. 2001). In the two nests for which 


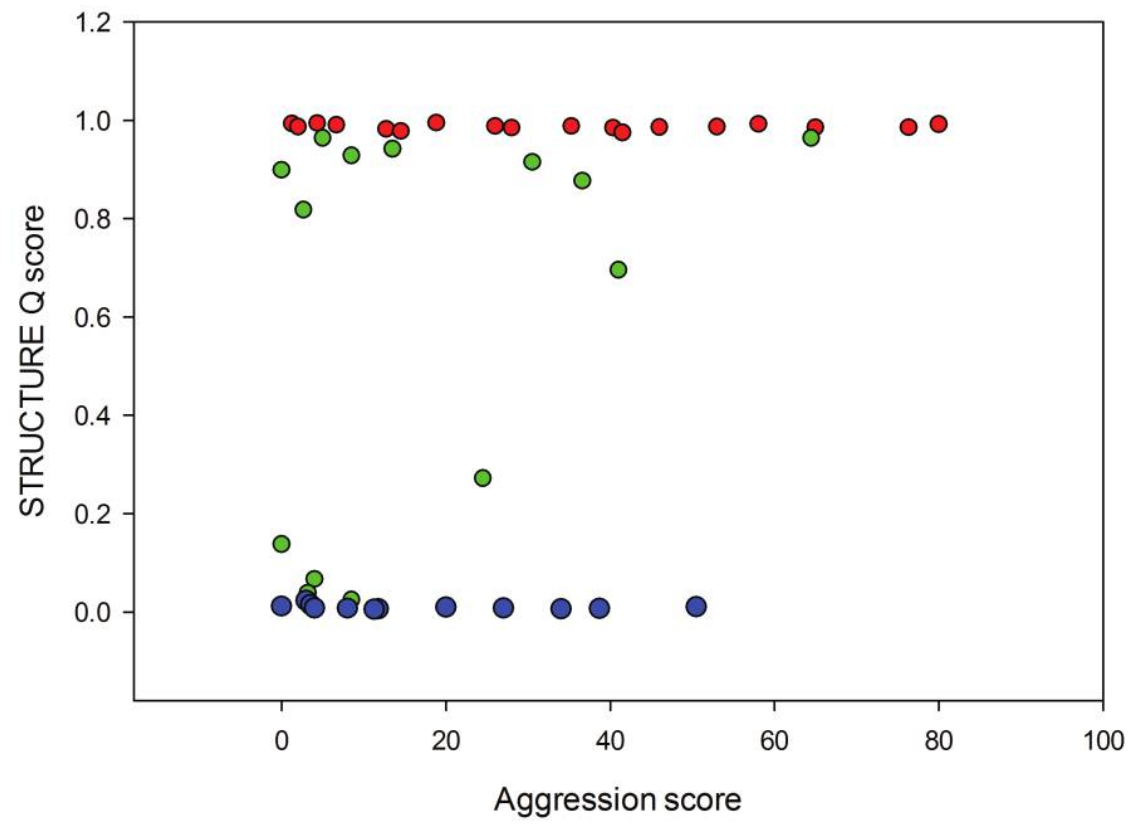

Figure 5: Mountain (blue circles) and western (red circles) bluebirds (Sialia currucoides and Sialia mexicana) differ in aggression, with western bluebirds having highly aggressive individuals that mountain bluebirds lack. Hybrid and introgressed individuals (green circles) were more similar to mountain bluebirds in their aggressive phenotype than to western bluebirds.

we had genetic samples, most of the offspring were from extrapair matings ( $75 \%$ and $66 \%$ of offspring were extrapair), and it appears that the females of both hybridizing pairs chose mountain bluebird males as their extrapair mates. In the UMC nest, we were able to identify a neighboring purebred mountain bluebird male as the extrapair father, and in the MVA nest we inferred that the extrapair mate was a purebred mountain bluebird, given that the offspring were a mixture of purebred extrapair and hybrid within-pair offspring (fig. 3). This pattern of extrapair mating suggests that female mountain bluebirds recognize and prefer males of their own species and pair with western bluebird males mainly for access to a nest cavity. In theory, this pattern of extrapair paternity in mixed pairs should select on male mate choice; however, such selection is likely to be weak because hybridization is so rare (only $0.23 \%$ of pairs were heterospecific). Importantly, because of the disparity between the two species in competitive ability, we would not expect female western bluebirds to similarly benefit from pairing with a mountain bluebird male during late stages of population colonization because in these late stages mountain bluebirds are typically breeding in either low-quality nest cavities or on the periphery of populations in suboptimal habitat (Duckworth et al. 2017). Thus, this study adds to a growing body of evidence that differences in aggression and competitive ability among parent species can strongly influence the direction of heterospecific pairing (Pearson and Rohwer 2000; Shurtliff et al. 2013; Robbins et al. 2014).
While differences in competitive ability likely influence the dynamics of hybridization, the observation that at least one of the heterospecific pairings involved individuals of hybrid origin complicates the story. In the UMC population, the female of the heterospecific pair was of hybrid origin, and she went on to pair in the following year with a different male that was also categorized as of hybrid origin. The implication of this observation is that once hybrids are produced, either they may selectively hybridize with each other or they are very nonselective in their choice of mates. It is unclear what criteria the hybrid female was using to select her social mate, as her first mate was very western-like in plumage and the second mate was more mountain-like. Thus, if hybrids do more often prefer each other, it may be based on behavioral rather than plumage characters. Alternatively, purebreds may generally avoid mating with hybrids, increasing the chances of hybrids pairing with one another. Either way, if assortative pairing among individuals of hybrid origin is common, it has important evolutionary implications because assortative mating among hybrids has been hypothesized as a route to rapid hybrid speciation in animals (Melo et al. 2009).

Hybridization between western and mountain bluebirds impacted expression of both morphology and behavior. We found that hybrids were more similar in structural traits to western bluebirds because they were smaller than mountain bluebirds but were more similar to mountain than western bluebirds in aggression. Western bluebirds are more aggressive than mountain bluebirds mainly because about one-third 
of individuals express a highly aggressive phenotype (Duckworth 2008) that mountain bluebirds and hybrids lack (Duckworth and Badyaev 2007; fig. 5). Thus, our results suggest that different types of traits assort differently in hybrids; not only does this mean that hybridization between these species has potentially important consequences for phenotypic evolution, it also makes this system a wealth of information for future studies of the assortment of behavioral and morphological traits.

It has long been recognized that heterospecific pairing more often occurs when one species is rare relative to the other (Hubbs 1955; Randler 2002), and there are numerous studies showing a strong link between hybridization and range expansion or habitat transitions where parental populations often come together in disproportionate numbers (Petit et al. 2003; Burgess et al. 2005; Rieseberg et al. 2007; Currat et al. 2008; Lepais et al. 2009). Because ecological succession is characterized by predictable transitions in the relative proportion of species over time, it shares many of the characteristics of these other transitions. However, unlike other examples of hybridization contexts, it is not limited to species boundaries and can occur throughout a species' range, making the dynamics of hybridization in the context of succession potentially quite different from other ecological contexts. Yet because the overlap of closely related taxa during succession is often brief, heterospecific mating may not be readily apparent, and so we suggest that this ecological context, which is widespread in nature, may be a frequently overlooked but potentially important context for hybridization.

\section{Acknowledgments}

We are grateful to D. Anderson and B. Anderson for allowing access to their property and to the late E. Davis for letting us know about a hybridizing pair on his nest box trail. M. Jacobson helped with DNA extraction and polymerase chain reaction, and numerous field technicians helped with data collection. We thank the Badyaev and Duckworth labs for their feedback on this project and are grateful to A. Badyaev for designing figure 1 . This work was supported by National Science Foundation grants DEB-918095 and DEB-1350107 to R.A.D. and a G. G. Simpson Fellowship to G.A.S.

\section{Literature Cited}

Abbott, R. J., J. K. James, R. I. Milne, and A. C. Gillies. 2003. Plant introductions, hybridization and gene flow. Philosophical Transactions of the Roval Societv B 358:1123-1132.

Abramoff, M. D., P. J. Magalhaes, and S. J. Ram. 2004. Image processing with ImageJ. Biophotonics International 11:36-42.

Aguillon, S. M., and R. A. Duckworth. 2015. Kin aggression and resource availability influence phenotype-dependent dispersal in a passerine bird. Behavioral Ecology and Sociobiology 69:625-633.

Anderson, E. 1949. Hybridization of the habitat. Evolution 2:1-9.
Anderson, E. C., and E. A. Thompson. 2002. A model-based method for identifying species hybrids using multilocus genetic data. Genetics 160:1217-1229.

Arnold, M. L. 1992. Natural hybridization as an evolutionary process. Annual Review of Ecology and Systematics 23:237-261.

Aylesworth, A. 1987. Mountain $\times$ western bluebird hybrids. Sialia 9:9-21.

Barton, N. H. 2001. The role of hybridization in evolution. Molecular Ecology 10:551-568.

Burgarella, C., Z. Lorenzo, R. Jabbour-Zahab, R. Lumaret, E. Guichoux, R. J. Petit, A. Soto, and L. Gil. 2009. Detection of hybrids in nature: application to oaks (Quercus suber and Q. ilex). Heredity 102:442-452.

Burgess, K. S., M. Morgan, L. Deverno, and B. C. Husband. 2005. Asymmetrical introgression between two Morus species (M. alba, M. rubra) that differ in abundance. Molecular Ecology 14:3471-3483.

Cadotte, M. W. 2007. Competition-colonization trade-offs and disturbance effects at multiple scales. Ecology 88:823-829.

Cadotte, M. W., D. V. Mai, S. Jantz, M. D. Collins, M. Keele, and J. A. Drake. 2006. On testing the competition-colonization tradeoff in a multispecies assemblage. American Naturalist 168:704-709.

Currat, M., M. Ruedi, R. J. Petit, and L. Excoffier. 2008. The hidden side of invasions: massive introgression by local genes. Evolution 62:1908-1920.

Duckworth, R. A. 2006a. Aggressive behaviour affects selection on morphology by influencing settlement patterns in a passerine bird. Proceedings of the Roval Society B 273:1789-95.

2006b. Behavioral correlations across breeding contexts provide a mechanism for a cost of aggression. Behavioral Ecology 17: 1011-1019.

. 2006c. Evolutionary ecology of avian behavior: from individual variation to geographic range shifts. PhD diss. Duke University, Durham, NC.

-2008. Adaptive dispersal strategies and the dynamics of a range expansion. American Naturalist 172(suppl.):S4-S17.

- 2012. Evolution of genetically integrated dispersal strategies. Pages 83-94 in J. Clobert, M. Baguette, T. G. Benton, and J. M. Bullock, eds. Dispersal ecology and evolution. Oxford University Press, Oxford.

-2014. Human-induced changes in the dynamics of species coexistence: an example with two sister species. Pages 181-191 in D. Gil and H. Brumm, eds. Avian urban ecology: behavioural and physiological adaptations. Oxford University Press, Oxford.

Duckworth, R. A., and A. V. Badyaev. 2007. Coupling of dispersal and aggression facilitates the rapid range expansion of a passerine bird. Proceedings of the National Academy of Sciences of the USA 104:15017-15022.

Duckworth, R. A., V. Belloni, and S. R. Anderson. 2015. Cycles of species replacement emerge from locally induced maternal effects on offspring behavior in a passerine bird. Science 374:875-877.

Duckworth, R. A., K. K. Hallinger, N. Hall, and A. L. Potticary. 2017. Switch to a novel breeding resource influences coexistence of two passerine birds. Frontiers in Ecology and Evolution 5:72, doi:10.3389 /fevo.2017.00072.

Duckworth, R. A., and L. E. B. Kruuk. 2009. Evolution of genetic integration between dispersal and colonization ability in a bird. Evolution 63:968-977.

Duckworth, R. A., and G. Semenov. 2017. Data from: Hybridization associated with cycles of ecological succession in a passerine bird. American Naturalist, Dryad Digital Repository, http://dx.doi.org /10.5061/dryad.6dm3c. 
Duckworth, R. A., and K. W. Sockman. 2012. Proximate mechanisms of behavioural inflexibility: implications for the evolution of personality traits. Functional Ecology 26:559-566.

Emms, S. K., and M. L. Arnold. 1997. The effect of habitat on parental and hybrid fitness: transplant experiments with Louisiana irises. Evolution 51:1112-1119.

Ferree, E. D., J. L. Dickinson, D. Kleiber, C. A. Stern, J. Haydock, M. T. Stanback, V. Schmidt, L. Eisenberg, and C. Stolzenburg. 2008. Development and cross-species testing of western bluebird (Sialia mexicana) microsatellite primers. Molecular Ecology Resources 8: 1348-1350.

Grant, P. R., and B. R. Grant. 1992. Hybridization of bird species. Science 256:193-197.

Guinan, J. A., P. A. Gowaty, and E. K. Eltzroth. 2000. Western bluebird (Sialia mexicana). Birds of North America 510:1-31.

Hubbs, C. L. 1955. Hybridization between fish species in nature. Systematic Zoology 4:1-20.

Hutto, R. L., and D. A. Patterson. 2016. Positive effects of fire on birds may appear only under narrow combinations of fire severity and time-since-fire. International Journal of Wildland Fire 25:1074-1085.

Jakobsson, M., and N. A. Rosenberg. 2007. Clumpp: a cluster matching and permutation program for dealing with label switching and multimodality in analysis of population structure. Bioinformatics 23:1801-1806.

Jansson, G., C.-G. Thulin, and Å. Pehrson. 2007. Factors related to the occurrence of hybrids between brown hares Lepus europaeus and mountain hares L. timidus in Sweden. Ecography 30:709-715.

Kearse, M., R. Moir, A. Wilson, S. Stones-Havas, M. Cheung, S. Sturrock, S. Buxton, et al. 2012. Geneious basic: an integrated and extendable desktop software platform for the organization and analysis of sequence data. Bioinformatics 28:1647-1649.

Larson, E. L., C. Guilherme Becker, E. R. Bondra, and R. G. Harrison. 2013. Structure of a mosaic hybrid zone between the field crickets Gryllus firmus and G. pennsylvanicus. Ecology and Evolution 3:985-1002.

Lepais, O., R. J. Petit, E. Guichoux, J. E. Lavabre, F. Alberto, A. Kremer, and S. Gerber. 2009. Species relative abundance and direction of introgression in oaks. Molecular Ecology 18:2228-2242.

Levin, S. A., and R. T. Paine. 1974. Disturbance, patch formation, and community structure. Proceedings of the National Academv of Sciences of the USA 71:2744-2747.

Mallet, J. 2005. Hybridization as an invasion of the genome. Trends in Ecology and Evolution 20:229-237.

Mallet, J., N. Besansky, and M. W. Hahn. 2016. How reticulated are species? BioEssavs 38:140-149.

Mayr, E. 1963. Animal species and evolution. Harvard University Press, Cambridge, MA.

Melo, M. C., C. Salazar, C. D. Jiggins, and M. Linares. 2009. Assortative mating preferences among hybrids offers a route to hybrid speciation. Evolution 63:1660-1665.

Miller, S. D., D. Dykes, and H. Polesky. 1988. A simple salting out procedure for extracting DNA from human nucleated cells. Nucleic Acids Research 16:1215.

Nielsen, E. E., L. A. Bach, and P. Kotlicki. 2006. HYBRIDLAB (version 1.0): a program for generating simulated hybrids from population samples. Molecular Ecology Notes 6:971-973.

Pearson, S. F., and S. Rohwer. 2000. Asymmetries in male aggression across an avian hybrid zone. Behavioral Ecology 11:93-101.

Petit, R. J., C. Bodénès, A. Ducousso, G. Roussel, and A. Kremer. 2003. Hybridization as a mechanism of invasion in oaks. New Phytologist 161:151-164.

Platt, W. J., and J. H. Connell. 2003. Natural disturbances and directional replacement of species. Ecological Monographs 73:507-522.
Power, H. W., and M. P. Lombardo. 1996. Mountain bluebird. Pages 121 in A. Poole and F. Gill, eds. The Birds of North America. Cornell Laboratory of Ornithology, Ithaca, NY.

Pritchard, J. K., M. Stephens, and P. Donelly. 2000. Inference of population structure using multilocus genotype data. Genetics 155:945-959.

Rand, D. M., and R. G. Harrison. 1989. Ecological genetics of a mosaic hybrid zone: mitochondrial, nuclear, and reproductive differentiation of crickets by soil type. Evolution 43:432-449.

Randler, C. 2002. Avian hybridization, mixed pairing and female choice. Animal Behaviour 63:103-119.

Rheindt, F. E., and S. V. Edwards. 2011. Genetic introgression: an integral but neglected component of speciation in birds. Auk 128:620-632.

Rhymer, J. M., and D. Simberloff. 1996. Extinction by hybridization and introgression. Annual Review of Ecology and Systematics 27:83-109.

Rieseberg, L. H., S. C. Kim, R. A. Randell, K. D. Whitney, B. L. Gross, C. Lexer, and K. Clay. 2007. Hybridization and the colonization of novel habitats by annual sunflowers. Genetica 129:149-165.

Robbins, T. R., L. E. Walker, K. D. Gorospe, S. A. Karl, A. W. Schrey, E. D. McCoy, and H. R. Mushinsky. 2014. Rise and fall of a hybrid zone: implications for the roles of aggression, mate choice, and secondary succession. Journal of Heredity 105:226-236.

Saab, V. A., R. E. Russell, and J. G. Dudley. 2007. Nest densities of cavitynesting birds in relation to postfire salvage logging and time since wildfire. Condor 109:97-108.

Schwenk, K., N. Brede, and B. Streit. 2008. Introduction. Extent, processes and evolutionary impact of interspecific hybridization in animals. Philosophical Transactions of the Roval Societv B 363:2805-2811.

Seehausen, O. 2004. Hybridization and adaptive radiation. Trends in Ecology and Evolution 19:198-207.

Senn, H. V., and J. M. Pemberton. 2009. Variable extent of hybridization between invasive sika (Cervus nippon) and native red deer (C. elaphus) in a small geographical area. Molecular Ecology 18: 862-876.

Shizuka, D., and J. L. Dickinson. 2005. Using molt limits to age western bluebirds. Lournal of Field Ornithology 76:193-196.

Shurtliff, Q. R., P. J. Murphy, J. D. Yeiter, and M. D. Matocq. 2013 Experimental evidence for asymmetric mate preference and aggression: behavioral interactions in a woodrat (Neotoma) hybrid zone. BMC Evolutionary Biology 13:1-13.

Taylor, S. A., T. A. White, W. M. Hochachka, V. Ferretti, R. L. Curry, and I. Lovette. 2014. Climate-mediated movement of an avian hybrid zone. Current Biology 24:671-676.

Vaha, J. P., and C. R. Primmer. 2006. Efficiency of model-based Bayesian methods for detecting hybrid individuals under different hybridization scenarios and with different numbers of loci. Molecular Ecology 15:63-72.

Veen, T., T. Borge, S. C. Griffith, S. Glenn-Peter, S. Bures, L. Gustafsson, and B. C. Sheldon. 2001. Hybridization and adaptive mate choice in flycatchers. Nature 411:45-50.

Wang, J., and A. W. Santure. 2009. Parentage and sibship inference from multilocus genotype data under polygamy. Genetics 181:1579-1594.

Yu, D. W., and H. B. Wilson. 2001. The competition-colonization trade-off is dead; long live the competition-colonization trade-off. American Naturalist 158:49-63.

\section{References Cited Only in the Online Appendixes}

Evanno, G., S. Regnaut, and J. Goudet. 2005. Detecting the number of clusters of individuals using the software STRUCTURE: a simulation study. Molecular Ecology 14:2611-2620.

Natural History Editor: Mark A. McPeek 